\title{
III-V-on-Silicon Photonic Transceivers for Radio-over-Fiber Links
}

\author{
K. Van Gasse, Student Member, IEEE, J. Van Kerrebrouck, Student Member, IEEE, \\ A. Abbasi, Member, IEEE, J. Verbist, Student Member, IEEE, G. Torfs, Member, IEEE, B. Moeneclaey, Student \\ Member, IEEE, G. Morthier, Senior Member, IEEE, X. Yin, Member, IEEE, J. Bauwelinck, Member, IEEE, \\ G. Roelkens, Member, IEEE
}

\begin{abstract}
The emergence of the fifth generation wireless networks (5G) and the Internet of Things drives the research on radio access networks (RANs). Future wireless networks aim to deliver a dramatic increase in bandwidth to an ever increasing amount of connected devices. To realize such a vision, advanced optical transceivers that link the base-station and the antennas will be required. In this work we present the first demonstration of an integrated silicon photonic radio-overfiber (RoF) transmitter and receiver, consisting of a directly modulated III-V-on-silicon distributed feedback laser and a Geon-Si waveguide photodiode co-integrated with a linear $\mathrm{SiGe}$ BiCMOS transimpedance amplifier respectively. Transmission of a high-spectral-efficiency Long-Term Evolution (LTE) $20 \mathrm{MHz}$ bandwidth $120 \mathrm{Mbit} / \mathrm{s}$ 64-QAM OFDM signal on a 3.5 and $5 \mathrm{GHz}$ carrier over $5 \mathrm{~km}$ of standard single mode fiber is demonstrated. We further demonstrate the transmission of 16 Gbps 16-QAM data on a $20 \mathrm{GHz}$ carrier over a $5 \mathrm{~km}$ link with a received error vector magnitude of $7 \%$. The use of silicon photonics provides the possibility of scaling, very much needed in the wireless communication networks of the future.
\end{abstract}

Index Terms-Silicon photonic transceivers, Radio-over-Fiber, Microwave Photonics, DFB lasers

\section{INTRODUCTION}

We are on the brink of a fully connected society, where everyone and everything is constantly exchanging information. Two pillars of this future society are the fifth generation wireless network $(5 \mathrm{G})$ and the Internet of Things. The goals for the $5 \mathrm{G}$ network are incredibly ambitious: delivering 10 Gbps connections to users, seamless connectivity, low-latency and a total data traffic increase of a 1000-fold. To achieve these ambitious goals, radio access networks (RANs) will need to be rethought. Future RANs will need to incorporate small cells, use of higher carrier frequencies and massive MIMO.

This work was partly supported by the European Commission in the framework of the 2020-ICT-2014-2 project Flex5Gware (Grant agreement no. 671563)

K. Van Gasse is with the Photonics Research Group, Department of Information Technology, Ghent Universityimec, Ghent 9052, Belgium (e-mail: kasper.vangasse@ugent.be).

J. Van Kerrebrouck, J. Verbist, B. Moeneclaey, G. Torfs, X. Yin and J. Bauwelinck are with IDLab, Department of Information Technology, Ghent University-imec, Ghent 9052, Belgium (email:_ joris.vankerrebrouck@ugent.be; jochem.verbist@ugent.be; bart.moeneclaey@ugent.be; guy.torfs@ugent.be; xin.yin@ugent.be; johan.bauwelinck@ugent.be).

A. Abbasi, H. Chen, G. Morthier and G. Roelkens are with the Photonics Research Group, Department of Information Technology, Ghent University-imec, Ghent 9052, Belgium (e-mail: amin.abbasi@ugent.be; hongtao.chen@ugent.be; geert.morthier@ugent.be; gunther.roelkens@ugent.be).
Especially in a cloud-RAN configuration, where the small cells are coordinated by a centralized base-station, radio-overfiber (RoF) links can play an essential role[1]. Given the the large number of links that would be needed in such a cloud RAN, a low-cost and scalable transceiver solution is needed. Integrated microwave photonics (IMWP) is the ideal technology to realize this [2].

Many promising IMWP functions and sub-systems have been demonstrated such as tunable delays, tunable filters and beamforming networks[3][4]. Most of these photonic integrated circuits (PICs) were realized in passive photonic technologies, using external active components. In a recent demonstration a monolithic integrated photonic microwave filter was realized on the InP platform [5], including the integration of laser sources, optical modulators and photodetectors, which is an important milestone. As a hybrid approach, III-V-on-silicon photonic integrated circuits can also be used for integrated microwave photonics, combining the low loss of silicon / silicon nitride waveguide circuits with the active functionality implemented in III-V semiconductors. This technology has shown promising results for agile microwave signal generation [6] [7] and highly linear modulators [8].

While different IMWP functions have been demonstrated, the important functionality of integrated analogue RoF transmitters (Tx) and receivers $(\mathrm{Rx})$ remains to be demonstrated. In this paper, we demonstrate the use of III-V-on-silicon Tx and silicon photonic receivers for RoF links. A III-V-on-silicon distributed feedback laser is used at the Tx side. For RoF links it is interesting to use a directly modulated laser instead of a continuous wave laser and a Mach-Zehnder Modulator (MZM), as the insertion loss of the external modulator is eliminated this way and the component count is reduced. At the receiver side high-bandwidth waveguide-coupled Geon-Si photodetectors are used, integrated with a linear SiGe BiCMOS transimpedance amplifier (TIA). The scalability and cost-effectiveness of such Tx and Rx PICs make them a great candidate for mass deployment in future RANs. We perform two link experiments using these silicon photonic transceivers, showcasing their potential for various waveforms. In a first experiment we transmit a high-spectral-efficiency LTE-type 64-QAM orthogonal frequency division multiplexing (OFDM) signal over $5 \mathrm{~km}$ single mode fiber at 3.5 and $5 \mathrm{GHz}$ carrier frequency. The quasi error free performance of these links attests to the linearity and low noise of the Tx and Rx. In a second experiment, using the same transmitter but a higher 
bandwidth receiver, we transmit a broadband 16 Gbps 16QAM data signal on a $20 \mathrm{GHz}$ carrier over a $5 \mathrm{~km}$ single mode fiber link, demonstrating that high bitrate transmission can be obtained using these integrated devices.

The remainder of this paper is divided in three main sections. In the first section we describe the use of the IIIV-on-silicon laser transmitter and Ge-on-silicon waveguide photodiode receiver (with integrated TIA) for the LTE link at 3.5 GHz and $5 \mathrm{GHz}$ carrier frequency. The positive impact of using the Ge-on-Si photodiode in avalanche mode is discussed. In the second section we describe the use of the same III-Von-silicon transmitter together with a higher bandwidth silicon photonic waveguide-coupled Ge-on-Si lateral p-i-n photodetector receiver (again with integrated TIA) for 16 Gbps 16QAM data transmission at $20 \mathrm{GHz}$ carrier frequency. These 16-QAM results are an extension of the content presented in [9]. In the final section the results are summarized and a conclusion is presented.

\section{3.5 GHz AND $5 \mathrm{GHz}$ 64-QAM OFDM RADIO-OVER-FIBER LINK}

\section{A. III-V-on-silicon transmitter}

The transmitter is a directly modulated III-V-on-silicon DFB laser. The laser is fabricated by divinylsiloxane-bisbenzocyclobutene (DVS-BCB) adhesive wafer bonding of an InP/AlGaInAs multi-quantum-well (MQW) epitaxial stack to a silicon photonic integrated circuit [10]. The gain section is defined in the bonded III-V material by contact lithography followed by a combination of wet and dry etching. The silicon PIC contains $400 \mathrm{~nm}$ thick silicon ridge waveguides with a $180 \mathrm{~nm}$ etch depth, planarized down to the silicon device layer using $\mathrm{SiO}_{2}$. Underneath the III-V gain material a DFB grating (grating period $245 \mathrm{~nm}$, grating duty cycle of $50 \%$, $340 \mu \mathrm{m}$ length) is defined in the silicon waveguide. The light is coupled from the InP gain section to the passive $\mathrm{Si}$ waveguide by tapering both the InP and $\mathrm{Si}$ waveguide, using $180 \mu \mathrm{m}$ long taper structures. The taper structure insures high coupling efficiency and low reflection. The taper structures are electrically isolated from the DFB laser and are DC biased at $5 \mathrm{~mA}$. The laser is connected to a fiber-to-chip grating coupler in the silicon. A schematic of the device together with a microscope image is shown in Fig. 1.

The III-V-on-silicon DFB laser was first characterized on a temperature-controlled stage and contacted using electrical probe needles. The series resistance of the device is $7 \mathrm{Ohm}$. The threshold current is $20 \mathrm{~mA}$ and the output power coupled to the silicon waveguide is approximately $3 \mathrm{~mW}$ for 100 $\mathrm{mA}$ bias current. The light is coupled from the Si waveguide into a cleaved single mode fiber with a fiber-to-chip grating coupler with a coupling efficiency of $-7 \mathrm{~dB}$. The laser shows excellent single mode behavior at $1570 \mathrm{~nm}$, with a side mode suppression ratio of $40 \mathrm{~dB}$. To determine the laser frequency response a small signal measurement was performed using a Keysight PNA-X $67 \mathrm{GHz}$ network analyzer. In Fig. 3 the response of the laser is shown for three different bias currents. It is clear that the bandwidth increases with increasing bias current, due to the shift of the relaxation oscillation resonance

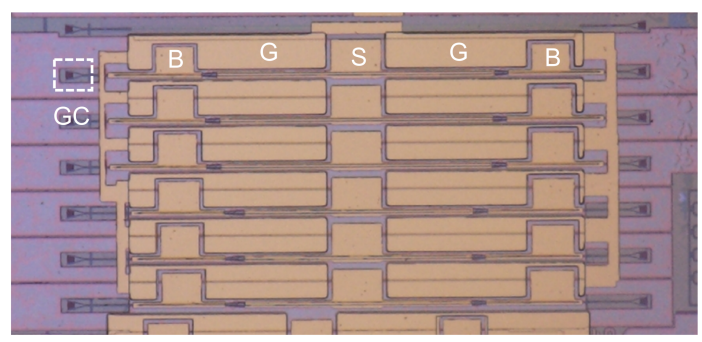

(a)

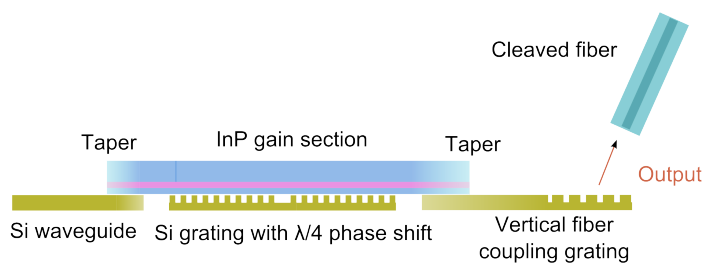

(b)

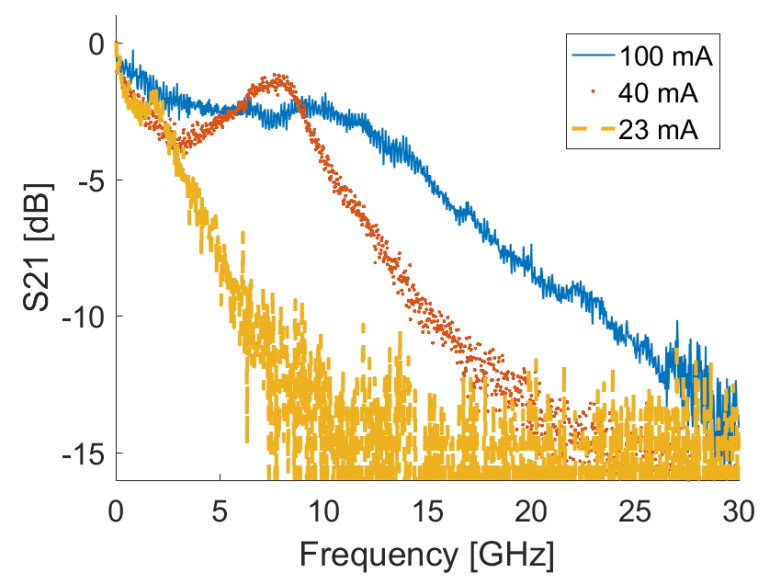

(c)

Fig. 1: (a) Microscope image of the Tx PIC showing an array of III-V-on-silicon DFB lasers. The vertical grating coupler is indicated with GC. The contact pads for the RF probe are indicated with $\mathrm{G}$ (Ground) and $\mathrm{S}$ (Signal). The contact pads for the tapers are indicated with B (Bias). (b) Schematic sideview of the III-V-on-silicon laser.(c) Small-signal response of the laser for different bias currents.

frequency. At an injection current of $100 \mathrm{~mA}$ a bandwidth above $10 \mathrm{GHz}$ is obtained.

\section{B. Ge-on-Si Receiver with integrated TIA}

The receiver consists of a $14 \mu \mathrm{m}$ long Ge-on-Si waveguidecoupled avalanche photodetector (APD) on a silicon photonic integrated circuit co-integrated with a TIA. The photodetector cross-section is shown in Fig. 2a. The photodetector consists of a $400 \mathrm{~nm}$ thick and $1000 \mathrm{~nm}$ wide Ge slab epitaxially grown on top of a n-doped $\mathrm{Si}$ waveguide. The Ge is p-doped and contacted with tungsten plugs. Due to this geometry and by optimizing the doping profile very high electrical fields 


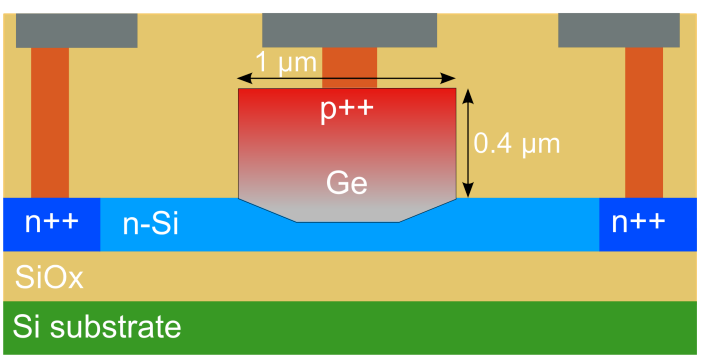

(a)

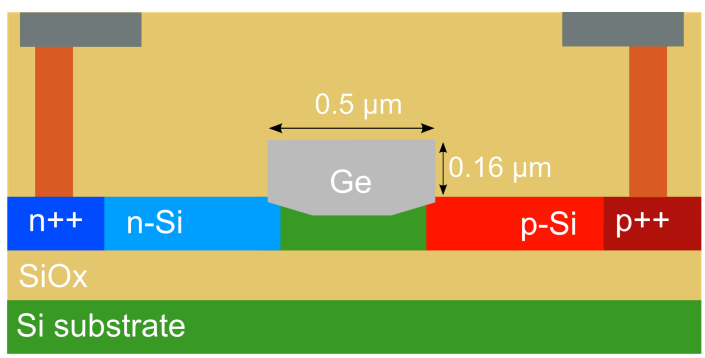

(b)

Fig. 2: (a) Cross section of the Ge-on-Si Avalanch Photodetector. The blue area denotes n-doped Si. (b) Cross section of the Ge-on-Si lateral p-i-n photodetector.

are created at low voltages in the germanium, allowing for avalanche operation at low bias voltages.

A gain-bandwidth product of $100 \mathrm{GHz}$ is achieved [11]. The PIC was co-integrated with the TIA on a printed circuit board using wire bonding. The TIA was designed in $0.13 \mathrm{~m} \mathrm{SiGe}$ BiCMOS technology and was designed for linear operation with an APD [12]. It consumes $200 \mathrm{~mW}$ in operation. The receiver has variable gain settings and the entire amplifier chain is designed for high linearity (no internal hard decisions) over the whole operating range.

\section{Link characterization}

While this $\mathrm{Tx} / \mathrm{Rx}$ combination was previously used for 10 Gbps non-return-to-zero digital data reception [13], the linearity or the influence of the avalanche gain on the complex modulation formats used in an analogue RoF link was not yet investigated. For the characterization of the link the III-V-onsilicon DFB laser was mounted on a temperature-controlled stage, kept at a constant temperature of 20C. The laser was contacted using a $40 \mathrm{GHz}$ bandwidth Cascade RF probe. The laser was biased using a bias-T and a stable current source. An Anritsu MS2692A was used as both a data signal generator (SG) and analyzer. The output of the SG was amplified by a $50 \mathrm{GHz}$ bandwidth SHF amplifier with $14 \mathrm{~dB}$ gain. The laser diode did not have a $50 \mathrm{Ohm}$ matching network and therefor part of the input signal is reflected to the amplifier. The optical output of the laser is $-3 \mathrm{dBm}$ in the fiber at 100 $\mathrm{mA}$ bias current due to the $7 \mathrm{~dB}$ loss of the grating coupler. To compensate these losses and to be able to vary the input power on the receiver without adjusting the laser bias current,

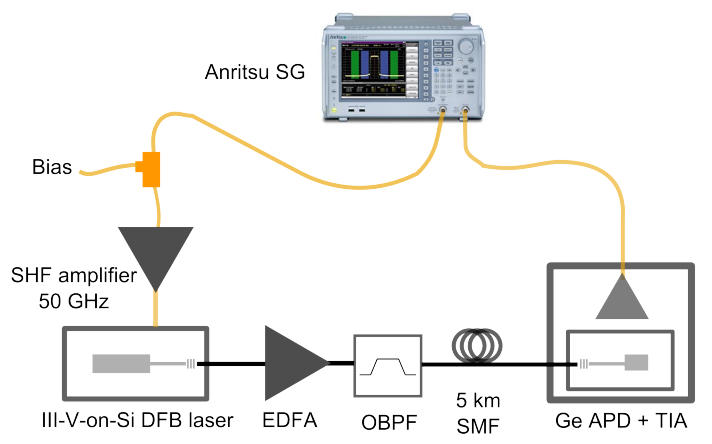

Fig. 3: Overview of the LTE link experiment setup.

a Keopsys Erbium Doped Fiber Amplifier (EDFA) and Santec OTF-350 optical filter was used. The EDFA was used in active current control to adjust the power output. The tunable optical filter (with an insertion loss of $4 \mathrm{~dB}$ ) was used to filter out the ASE. The optical signal was coupled onto the receiver PIC containing the Ge-on-Si PD, again incurring 7dB loss. A schematic of the measurement setup is shown in Fig. 3 . It is important to note that the EDFA is only necessary to investigate the signal quality as a function of optical input power for the receiver. As will be discussed below, no optical amplification is needed to achieve an rms EVM lower than $1.5 \%$.

First, the linearity of the link was measured using a twotone third-order intercept point (IP3) measurement. This measurement was performed using the Anritsu MS2692A both as SG and spectrum analyzer. The bias current of the laser was $100 \mathrm{~mA}$. The waveguide-coupled optical power received by the Ge photodiode was $-9 \mathrm{dBm}$. The measurement was performed with two tones spaced $5 \mathrm{MHz}$ at both 3.5 and $5 \mathrm{GHz}$. The measurement results are presented in Fig. 4. For a carrier frequency of $3.5 \mathrm{GHz}$ we find a spurious free dynamic range (SFDR) of $90.2 \mathrm{~dB} \cdot \mathrm{Hz}^{2 / 3}$, while for $5 \mathrm{GHz}$ the SFDR degrades slightly by $1.4 \mathrm{~dB}$ which is consistent with the low-frequency roll-off shown in Fig. 1c. This is worse than current state-of-the-art MZM based links [15]. However, the link experiment discussed below proves that the linearity is sufficient for error-free LTE-signal transmission. At 3.5 and $5 \mathrm{GHz}$ carrier frequencies, the third-order input intercept point (IIP3) occurs for $19.5 \mathrm{dBm}$ and $22 \mathrm{dBm}$ of input power respectively.

In a first transmission experiment a $20 \mathrm{MHz}$ bandwidth $120 \mathrm{Mbit} / \mathrm{s}$ 64-QAM OFDM signal, which corresponds to a LTE compliant test signal, was transmitted on a $3.5 \mathrm{GHz}$ carrier. Measurements were done both in an optical back-toback configuration and using a $5 \mathrm{~km}$ standard single mode fiber (SMF) link. The photodiode was used in p-i-n mode using $2 \mathrm{~V}$ reverse bias. An electronic back-to-back measurement, without electro-optic conversion gave a root mean square (rms) EVM of $0.6 \%$. When the signal was transmitted over 50 $\mathrm{m}$ of optical fiber the rms EVM degraded to $1.4 \%$. When the length of the fiber span is increased to $5 \mathrm{~km}$ the EVM degraded further to $1.7 \%$. This still corresponds to error-free operation. At $5 \mathrm{GHz}$ carrier frequency, an electric back-to-back measurement gave an rms EVM of $0.6 \%$. Optical transmission 


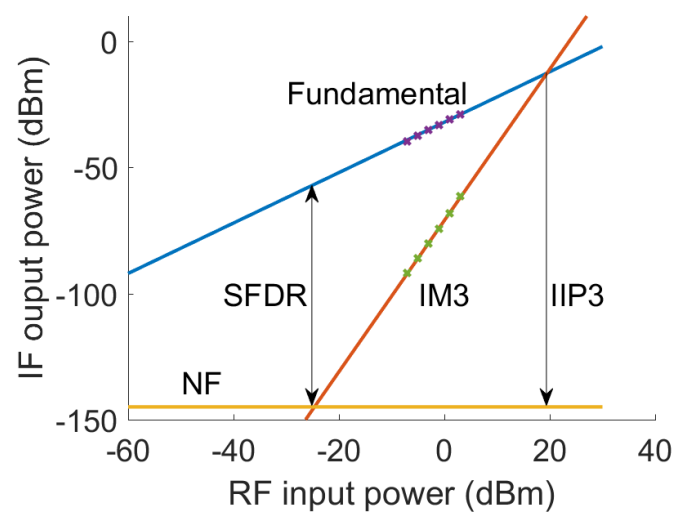

(a)

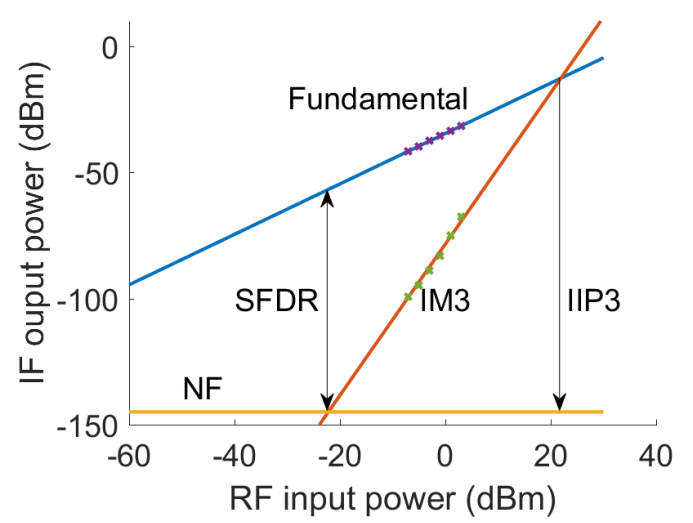

(b)

Fig. 4: IP3 measurement of the link for a carrier frequency of (a) $3.5 \mathrm{GHz}$ and (b) $5 \mathrm{GHz}$. NF: noise floor, IM3: intermodulation, IIP3: input intercept point.

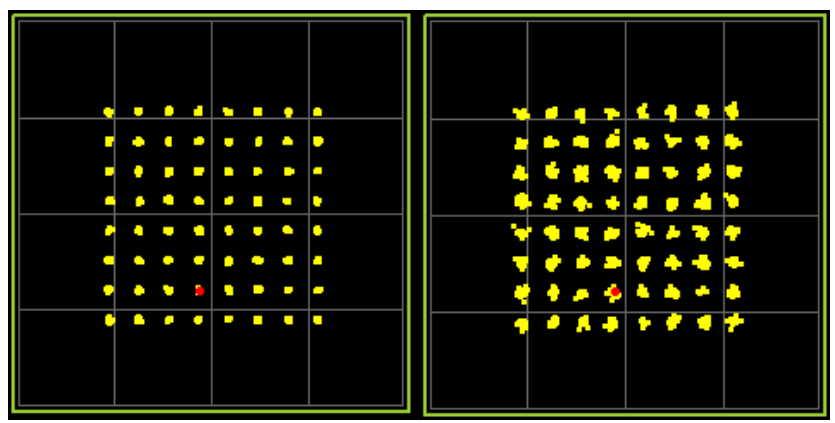

(a)

(b)

Fig. 5: Received constellation diagrams of a 64-QAM OFDM signal (of a representative subcarrier) for a carrier frequency of (a) $3.5 \mathrm{GHz}$ and (b) $5 \mathrm{GHz}$.

over $5 \mathrm{~km}$ of SMF showed a good signal quality, however the EVM degraded to $3.7 \%$ (resulting in a bit error ratio (BER) of approximately $10^{-8}$ ). The 64-QAM constellations of both signals (for a representative sub-carrier) can be found in Fig. 5.

To analyze the impact of using the photodetector in avalanche mode, we studied the photocurrent and EVM as

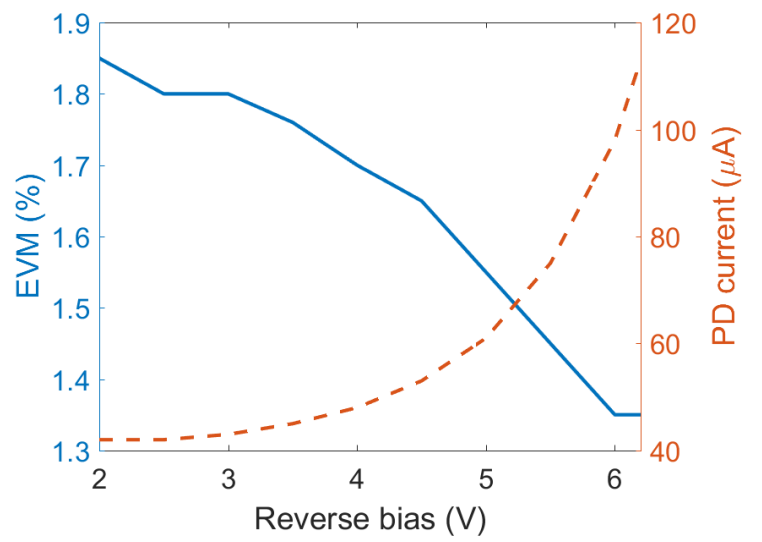

Fig. 6: The rms EVM and APD current of a $3.5 \mathrm{GHz}$ carrier 64-QAM OFDM signal transmitted over $5 \mathrm{~km}$ SMF as function of the reverse bias of the APD.

function of the reverse bias on the photodetector for a 5 $\mathrm{km}$ link length. At $2 \mathrm{~V}$ reverse bias the PD functions as a standard p-i-n detector with a responsivity of $0.6 \mathrm{~A} / \mathrm{W}$. To achieve avalanche amplification a reverse bias greater than 4 $\mathrm{V}$ is needed. Maximum amplification $(\mathrm{M}=2.7)$ is found for a reverse bias of $6.2 \mathrm{~V}$. From the graph in Fig. 6 (using an average optical input power on the detector of $-11.5 \mathrm{dBm}$ ) we see strong increase in the photocurrent beyond $4 \mathrm{~V}$ reverse bias. At the same time the EVM decreases, which means that the signal is amplified without the excess noise deteriorating the signal quality. Since the increase in reverse bias only causes a minute increase in power consumption compared to the power consumption of the laser or an optical amplifier, it provides a very power-efficient gain mechanism, allowing longer amplifier free link-spans.

In a second measurement, we studied the signal quality as function of optical input power, for the PD in p-i-n and APD mode. The RF power provided by the SG was in this case fixed to $-16 \mathrm{dBm}$, and the link length was $5 \mathrm{~km}$. We see that for higher optical input powers the signal quality improves and the EVM drops below 3\%, corresponding to error-free operation. For low optical input power the signal quality is substantially better in APD mode. As the optical power increases the rms EVM of the $p-i-n$ and APD mode converge. This shows that we can improve the sensitivity of the receiver by using the APD mode without compromising the performance at higher input power levels. This also illustrates that an EDFA is not necessary to achieve error-free transmission, even using the non-optimized fiber-to-chip grating couplers implemented in the Tx and Rx. Without EDFA (resulting in $-10 \mathrm{dBm}$ received optical power) a $4 \mathrm{~dB}(7 \mathrm{~dB})$ margin is obtained in $\mathrm{p}-\mathrm{i}-\mathrm{n}$ (APD) mode. Using high-efficiency fiber-chip interfaces, e.g. edge couplers with $1 \mathrm{~dB}$ insertion loss [14], the link budget would be further increased allowing passive optical splitting in antenna arrays without optical amplification.

Furthermore, we investigated the signal quality as a function of the RF input power provided by the SG, using the photodetector in avalanche mode $(-6.2 \mathrm{~V})$ and using an average optical input power on the detector of $70 \mathrm{uW}$ (link length of $5 \mathrm{~km}$ ). 


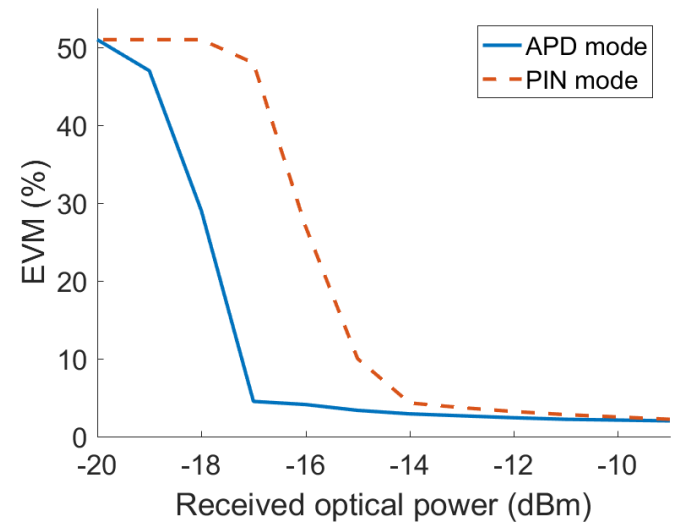

Fig. 7: The rms EVM as function of the optical power received by the Ge-on-Si PD. The RF power at the SG was kept constant at $-16 \mathrm{dBm}$.

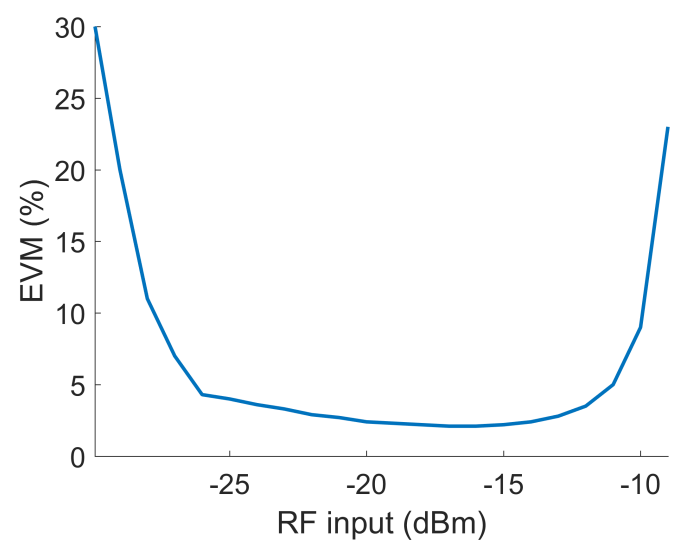

Fig. 8: The rms EVM as function of the RF power provided by the signal generator to the laser. The optical power received by the Ge-on-Si PD was $-11.5 \mathrm{dBm}$ and the PD was operated in APD mode at $6.2 \mathrm{~V}$ reverse bias.

This measurement shows we have a wide range of RF input powers delivering an EVM below $5 \%$. The increase in EVM for higher input powers can be understood, as overdriving the laser greatly compromises the linearity. When the driving signal is too low in power the SNR becomes insufficient.

\section{16 GBPS 16-QAM LINK AT 20 GHZ CARRIER FREQUENCY}

\section{A. III-V-on-silicon transmitter}

The same III-V-on-silicon transmitter as in the LTE experiment is used for the link at 16 Gbps 16-QAM link at $20 \mathrm{GHz}$ carrier frequency. The laser is biased at $100 \mathrm{~mA}$. While the carrier frequency is beyond the $3 \mathrm{~dB}$ modulation bandwidth of the laser, still good signal transmission can be obtained, through proper equalization (partly by the peaking of the receiver response and by using a finite impulse response (FIR) equalizer, as will be demonstrated below.

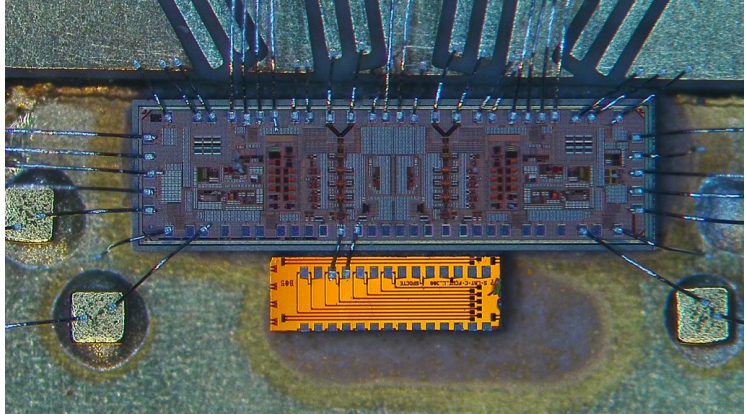

Fig. 9: A microscope image of the silicon photonic receiver: the Ge-on-Si photodetector (small die) wirebonded to the SiGe BiCMOS TIA (large die). [9]

\section{B. Ge-on-Si Receiver with integrated TIA}

Similar to the LTE experiment a waveguide-coupled Ge-on$\mathrm{Si}$ photodetector co-integrated with a SiGe BiCMOS TIA was used as receiver. However, in this receiver a $14 \mu \mathrm{m}$ long lateral p-i-n photodetector was used [16]. The photodetector cross section is shown in Fig. 2b. The photodetector consists of a 0.5 $\mu \mathrm{m}$ wide and $0.16 \mu \mathrm{m}$ thick germanium slab epitaxially grown on top of a $220 \mathrm{~nm}$ thick Si waveguide. A lateral p-i-n structure is defined with the p-contact and n-contact both on the silicon. To achieve optimal coupling between the Si waveguide and Ge absorber, a poly-Si optical taper is used. The responsivity for a bias voltage of $-1 \mathrm{~V}$ and a wavelength of $1550 \mathrm{~nm}$ is $0.72 \mathrm{~A} / \mathrm{W}$. The small-signal bandwidth of the device at a $-1 \mathrm{~V}$ bias exceeds $50 \mathrm{GHz}$. The TIA was fabricated using a $0.13 \mu \mathrm{m}$ SiGe BiCMOS process. It consumes approximately $180 \mathrm{~mW}$ and needs a $2.5 \mathrm{~V}$ supply. A more detailed description of the TIA can be found in [17]. Wirebond-based co-integration was used. A microscope picture of the silicon photonic receiver cointegrated with the TIA on a high-speed printed circuit board is shown in Fig. 9.

The small signal electro-optical response of the receiver is shown in Fig. 10, for $-2 \mathrm{dBm}$ of waveguide-coupled optical power. The response shows slight peaking and a $3 \mathrm{~dB}$ bandwidth of approximately $20 \mathrm{GHz}$. This peaking was designed in order to partially compensate for the limited bandwidth of the III-V-on-silicon laser. The bandwidth is fully determined by the TIA as the Si-on-Ge PD has a flat response up to $50 \mathrm{GHz}$.

To asses the linearity of the receiver an IP3 measurement was performed at $20 \mathrm{GHz}$ using the small-signal set-up. The PNA-X was used to generate two tones spaced $5 \mathrm{MHz}$ centered around $20 \mathrm{GHz}$ and the strength of the intermodulation distortion ( $2 f_{1}-f_{2}$ tone) was measured. The result of the IP3 measurement is shown in Fig. 11. The SFDR is $81 \mathrm{~dB} \cdot \mathrm{Hz}^{2 / 3}$ for a photocurrent of $450 \mu \mathrm{A}$.

\section{RoF Link demonstration}

As with the LTE link, the III-V-on-Silicon laser was placed on a temperature controlled stage and kept at a constant temperature of $20{ }^{\circ} \mathrm{C}$. It was again contacted with a 40 $\mathrm{GHz}$ Cascade RF probe and biased using a Keithley current source and bias-T. To generate the high bandwidth data signal a Keysight M8195A Arbitrary Waveform Generator 


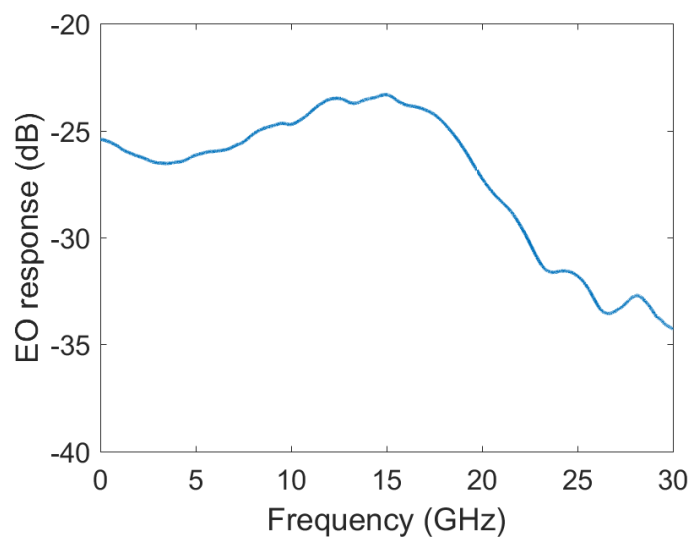

Fig. 10: Electro-optical response of the receiver (Ge receiver and SiGe BiCMOS TIA on a high-speed printed circuit board). [9]

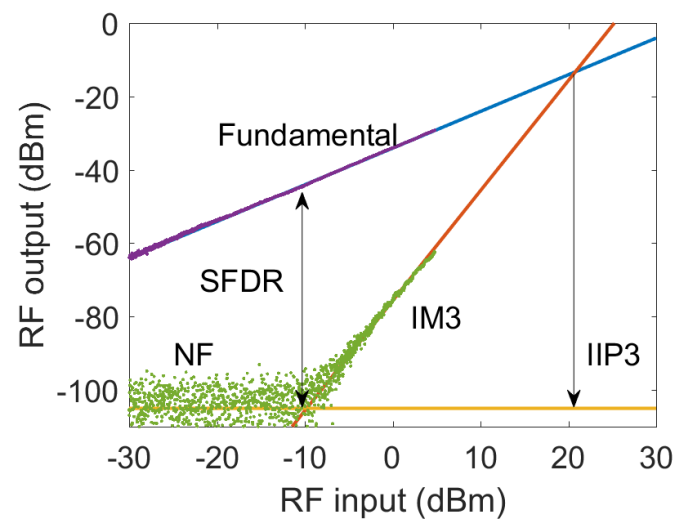

Fig. 11: IP3 measurement of the Ge-on-Si lateral p-i-n photodiode with TIA. The dots represent measured values, the full lines are extrapolated values. NF: noise floor, IM3: intermodulation, IIP3: input intercept point.

was used . Again the output of the laser is amplified with an EDFA to compensate the chip-to-fiber coupling losses. In this experiment $5 \mathrm{~km}$ standard $\mathrm{SMF}$ was used, before coupling to the silicon photonic receiver. The output of the receiver was captured by a $80 \mathrm{GSa} / \mathrm{s}$ real-time oscilloscope (Keysight DSAZ634A). An overview of the measurement setup is shown in Fig. 12. A series of transmission experiments was performed, where the symbol rate of a 16-QAM signal at $20 \mathrm{GHz}$ carrier frequency was increased from 1 to 5 Gbaud. For each transmission a $2^{9}-1$ symbols long pseudo random bit sequence (PRBS) was used. The received $1 \mathrm{GBd}$ signal was equalized online and found to have an error vector magnitude (EVM) of $5.7 \%$. The EVM was also determined online using the native vector signal analysis (VSA) software. To determine the BER, the unequalized waveform of $10^{7}$ symbols was saved and loaded into Matlab. There the measured single carrier signal is directly down mixed to a baseband I/Q signal. To obtain the symbol sample time of the I/Q signal, the Gardner algorithm was used [18]. A finite impulse response equalizer was implemented to reduce the Inter Symbol Interference

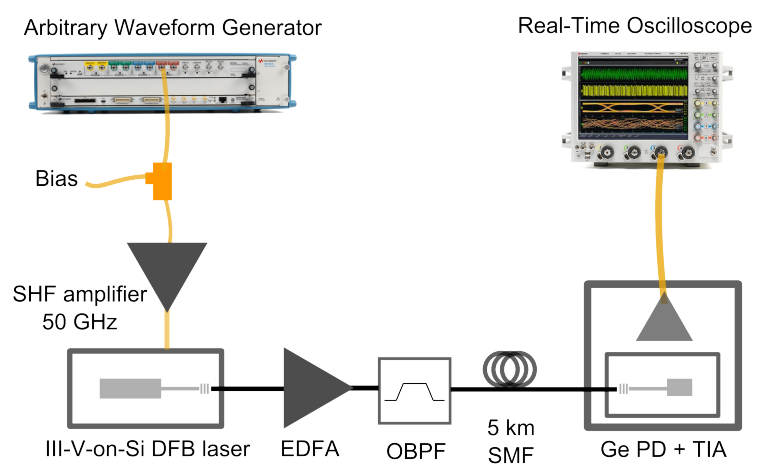

Fig. 12: Schematic of the $20 \mathrm{GHz}$ carrier transmission measurement set-up.

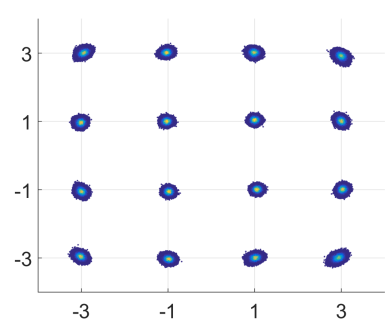

(a) $16 \mathrm{Gbps}(\mathrm{B} 2 \mathrm{~B})$

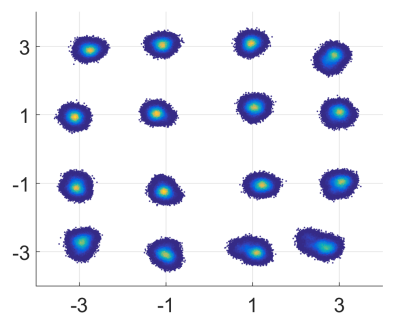

(c) 8 Gbps $(5 \mathrm{~km})$

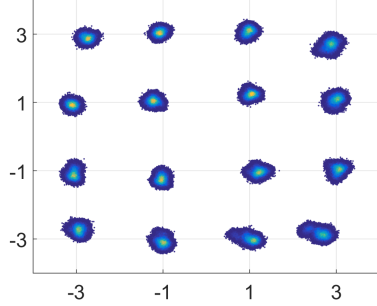

(b) 4 Gbps $(5 \mathrm{~km})$

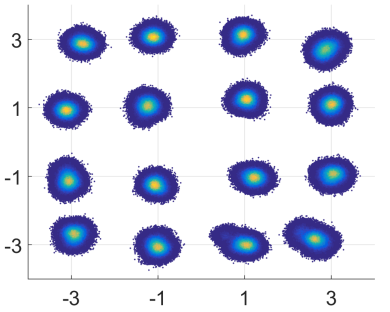

(d) 16 Gbps $(5 \mathrm{~km})$
Fig. 13: Constellation diagrams of $10^{6}$ 16-QAM symbols on a $20 \mathrm{GHz}$ carrier transmitted (a) electrically back-to-back and (b-d) over $5 \mathrm{~km}$ of SMF.

(ISI). A constellation of the equalized 1 Gbaud (4 Gbps) signal, based on $10^{6}$ received symbols, is shown in Fig. $13 \mathrm{~b}$.

The equalized transmission was found to be error free for a series of $10^{7}$ symbols, resulting in a BER below $2.5 \cdot 10^{-8}$. When no equalization was used, a BER of $5 \cdot 10^{-5}$ was found. We then further investigated the evolution of the online determined EVM as a function of the symbol rate of the 16QAM signal on a $20 \mathrm{GHz}$ carrier. This is shown in Fig. 14. A steady increase of the EVM up to $4 \mathrm{GBd}$ is observed, after which it sharply increases to $10 \%$ at $5 \mathrm{GBd}$. An offline analysis of the BER for 2 and 4 GBd was also performed by error counting. This gave a BER of $6 \cdot 10^{-7}$ for the $2 \mathrm{GBd}$ data and $3 \cdot 10^{-6}$ for the $4 \mathrm{GBd}$ data. The constellation of the 2 and 4 GBd transmission is also shown in Fig. 13b and 13c respectively. As a reference the constellation of an electrical $4 \mathrm{GBd}$ back-to-back measurement is shown in Fig. 13a.

These results show that the silicon photonic transmitter and 


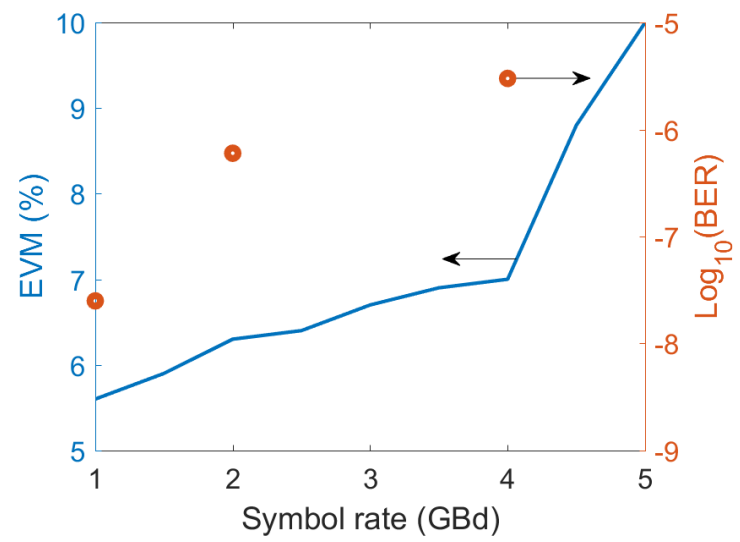

Fig. 14: The Error Vector Magnitude (EVM) and Bit Error Rate (BER) as function of the symbol rate, for a 16-QAM signal on a $20 \mathrm{GHz}$ carrier. The BER was determined by offline error counting.

receiver can also be used to transmit a high data-rate complex modulation format data on a high frequency carrier. Operation in the $28 \mathrm{GHz}$ frequency window - which is a frequency band of great interest for 5G wireless networks [19]- is within reach, as higher bandwidth TIAs (or narrow-bandwidth TIAs which peak at $28 \mathrm{GHz}$ ) are definitely feasible [20] and IIIV-on-Silicon DFB lasers with a bandwidth of $34 \mathrm{GHz}$ have been demonstrated[21]. Further integrating the laser with a matching circuit or dedicated driver could improve the channel quality.

\section{CONCLusions}

We have demonstrate the feasibility of using silicon photonics based transceivers for analogue radio-over-fiber links. Both a scenario in which a high spectral efficiency LTE signal is to be transmitted (requiring sufficient linearity of the link for transmitting the 64-QAM OFDM signal) and a scenario in which a high bitrate (16 Gbps 16-QAM) signal is transmitted is discussed. In both cases a directly modulated III-V-on-silicon laser is used, which simplifies the transmitter architecture. The use of silicon photonics provides the possibility of scaling, very much needed in the wireless communication networks of the future.

\section{REFERENCES}

[1] D C. Liu, J. Wang, L. Cheng, M. Zhu and G. K. Chang, "Key MicrowavePhotonics Technologies for Next-Generation Cloud-Based Radio Access Networks," J. Lightw. Technol., vol. 32, no. 20, pp. 3452-3460, 2014.

[2] S. Iezekiel, "Integrated microwave photonics: a key enabling technology for radio-over-fiber," Broadband Access Communication Technologies XI, Vol. 10128, pp. 1012803, Jan. 2017.

[3] D. Marpaung, C. Roeloffzen, R. Heideman, A. Leinse, S. Sales, J. Capmany, "Integrated microwave photonics." Laser Photonics Reviews, vol. 7, no. 4, pp.506-538, 2013.

[4] D. Pérez, I. Gasulla and J. Capmany, "Integrated microwave photonics," 2016 IEEE Photonics Conference (IPC), Waikoloa, HI, pp. 5-6, 2016.

[5] J. Fandino, P. Muñoz, D. Doménech, J. Capmany, "A monolithic integrated photonic microwave filter," Nature Photonics, vol. 11, no. 2, pp. 124-129, 2017.

[6] J. E. Bowers, T. Komljenovic, J. Hulme, M. Davenport and C. Zhang, "Integrated photonics for MWP," 2016 IEEE Photonics Conference (IPC), Waikoloa, HI, 2016, pp. 1-2.
[7] J. C. Hulme et al., "Fully integrated heterodyne microwave generation on heterogeneous silicon-III/V," 2016 IEEE International Topical Meeting on Microwave Photonics (MWP), Long Beach, CA, 2016, pp. 336-339.

[8] Chong Zhang, Paul A. Morton, Jacob B. Khurgin, Jon D. Peters, and John E. Bowers, "Ultralinear heterogeneously integrated ring-assisted MachZehnder interferometer modulator on silicon," Optica, Vol. 3, no. 12, pp. 1483-1488, 2016

[9] K. Van Gasse, J. van Kerrebrouck, A. Abbasi, G. Torfs, J. Bauwelinck and G. Roelkens, "16 Gbps RoF link at 20 ghz carrier frequency using a silicon photonics transmitter and receiver," 2017 International Topical Meeting on Microwave Photonics (MWP), Beijing, 2017, pp. 1-4.

[10] G. Roelkens et al., "III-V-on-silicon photonic devices for optical communication and sensing," Photonics (invited), vol. 2, no. 3, pp.969-1004, 2015

[11] H. Chen, J. Verbist, P. Verheyen, P. De Heyn, G. Lepage, J. De Coster, P. Absil, X. Yin, J. Bauwelinck, J. Van Campenhout, G. Roelkens, ”High sensitivity $10 \mathrm{~Gb} / \mathrm{s}$ Si photonic receivers based on a low-voltage waveguide coupled Ge avalanche photodetector," Opt. Express, vol. 23, no. 2, pp.815822,2015

[12] X. Yin, B. Moeneclaey, X. Qiu, J. Verbrugghe, K. Verheyen, J. Bauwelinck, J. Vandewege, M. Achouche, and Y. Chang, "A 10Gb/s APD-based linear burst-mode receiver with $31 \mathrm{~dB}$ dynamic range for reach-extended PON systems", Opt. Express, vol. 20, no. 26, pp. B462B469, 2012.

[13] A. Abbasi, B. Moeneclaey, X. Yin, J. Bauwelinck, G. Roelkens and G. Morthier, "10-/28-Gb Chirp Managed 20-km Links Based on Silicon Photonics Transceivers," IEEE Photon. Technol. Lett., vol. 29, no. 16, pp. 1324-1327, Aug. 2017.

[14] B. Ben Bakir et al., "Low-Loss $(<1 \mathrm{~dB})$ and Polarization-Insensitive Edge Fiber Couplers Fabricated on 200-mm Silicon-on-Insulator Wafers," IEEE Photon. Technol. Lett., vol. 22, no. 11, pp. 739-741, 2010.

[15] S. Li, X. Zheng, H. Zhang and B. Zhou, "Highly Linear Radio-OverFiber System Incorporating a Single-Drive Dual-Parallel MachZehnder Modulator," IEEE Photon. Technol. Lett., vol. 22, no. 24, pp. 1775-1777, 2010.

[16] H. Chen, P. Verheyen, P. De Heyn, G. Lepage, J. De Coster, S. Balakrishnan, P. Absil, W. Yao, L. Shen, G. Roelkens, J. Van Campenhout,"$1 \mathrm{~V}$ bias $67 \mathrm{GHz}$ bandwidth Si-contacted germanium waveguide pin photodetector for optical links at $56 \mathrm{Gbps}$ and beyond," Opt. Express, vol. 24, no.5, pp.4622-4631, 2016

[17] B. Moeneclaey et al., "Design and Experimental Verification of a Transimpedance Amplifier for $64 \mathrm{~Gb} / \mathrm{s}$ PAM-4 Optical Links," J. Lightw. Technol., vol. PP, no. 99, pp. 1-1. 2017

[18] F. Gardner "A BPSK/QPSK Timing-Error Detector for Sampled Receivers" IEEE Trans. Comm., vol. 34, no. 5, pp. 423-429, May 1986.

[19] H. Tan, W. Li, T. Wang, J. Fang and Z. Feng, "The analysis on the candidate frequency bands of future mobile communication systems," China Communications, vol. 12, pp. 140-149, 2015.

[20] I. García López, P. Rito, A. Awny, M. Ko, D. Kissinger and A. C. Ulusoy, "A DC-75-GHz Bandwidth and $54 \mathrm{~dB} \Omega$ Gain TIA With 10.9 $\mathrm{dB} \sqrt{\mathrm{Hz}}$ in 130-nm SiGe:C BiCMOS," IEEE Microwave and Wireless Components Letters, vol. 28, no. 1, pp. 61-63, Jan. 2018.

[21] A. Abbasi, B. Moeneclaey, J. Verbist, X. Yin, J. Bauwelinck, G.H. Duan, G. Roelkens, G. Morthier, "Direct and electro-absorption modulation of a III-V-on-silicon DFB laser at 56 Gbps," IEEE Journal of Selected Topics in Quantum Electronics, p.1501307 (7 pages) (2017) 